\title{
Biópsia hepática com agulha tru-cut guiada por videolaparoscopia em caprinos
}

\author{
[Videolaparoscopic guided hepatic biopsy with tru-cut needle in goats] \\ A.L.L. Duarte ${ }^{1}$, J.W. Cattelan ${ }^{2 *}$, M.B. Bezerra ${ }^{3}$, W.R.R. Vicente ${ }^{2}$, M.F. Cordeiro ${ }^{1}$ \\ ${ }^{1}$ Aluno de pós-graduação - FCAV-UNESP - Jaboticabal, SP \\ ${ }^{2}$ Faculdade de Ciências Agrárias e Veterinárias - UNESP \\ Via de Acesso Prof. Paulo Donato Castellane s/n \\ 14884-900 - Jaboticabal, SP \\ ${ }^{3}$ Universidade Federal Rural do Semi-Árido - Mossoró, RN
}

\begin{abstract}
RESUMO
Descreve-se a técnica de biópsia hepática com agulha tru-cut guiada por videolaparoscopia em 12 caprinos machos, castrados, hígidos, sem raça definida, distribuídos em dois grupos (G): G1, com cinco animais de 12 meses e G2, com sete de seis meses de idade. O procedimento foi realizado sob anestesia geral intravenosa com o animal em decúbito lateral esquerdo. O pneumoperitônio e a laparoscopia foram procedidos no flanco direito, aproximadamente a $10 \mathrm{~cm}$ ventral aos processos transversos das vértebras lombares. A agulha tru-cut foi introduzida no $11^{\circ}$ espaço intercostal direito, a aproximadamente $12 \mathrm{~cm}$ ventral à coluna vertebral, para punção e remoção de fragmento do lobo hepático direito. O tempo operatório médio foi de 23 minutos e cinco segundos. A hemorragia causada pela perfuração hepática cessou em dois minutos em $75 \%$ dos animais e em três minutos, nos $25 \%$ restantes. Nas avaliações clínicas feitas no pré-jejum e às 24,48 e 72 horas após a biópsia, não foram observadas alterações $(\mathrm{P}>0,05)$ da temperatura retal, das frequências cardíaca e respiratória e dos movimentos rumenais nos dois grupos. A biópsia hepática com agulha tru-cut guiada por videolaparoscopia foi considerada eficaz para uso em caprinos, permitindo a obtenção de fragmentos hepáticos suficientes para exame histológico.
\end{abstract}

Palavras-chave: caprino, agulha de biópsia, fígado, laparoscopia

\begin{abstract}
The videolaparoscopic guided hepatic biopsy with tru-cut needle is described in 12 healthy, no defined breed, castrated male goats. Animals were distributed in two groups: $G 1$ (n=5) 12-month-old animals; and G2 (n=7) six-month-old animals. The procedure was performed with the animal in left lateral recumbency and under total intravenous anesthesia. Pneumoperitoneum and laparoscopy were performed in the right flank, approximately $10 \mathrm{~cm}$ ventral to the transverse processes of the lumbar vertebrae. The tru-cut needle was inserted into the right eleventh intercostal space, around $12 \mathrm{~cm}$ ventral to the spinal column, for punching and removal of a fragment from the right hepatic lobe. The average time spent in the surgical procedure was 23 minutes and five seconds. The hemorrhage caused by the liver biopsy ceased in two minutes (75\%) and in three minutes in the other animals (25\%). In the clinical evaluations performed during the pre-fasting period and 24, 48, and 72 hours after the biopsy procedure, neither group presented alterations $(P>0.05)$ evaluated by rectal temperature; and cardiac, respiratory, and rumen frequencies. The videolaparoscopic guided hepatic biopsy with tru-cut needle was considered efficacious to be used in goats, providing hepatic tissue fragments suitable for histological examination.
\end{abstract}

Keywords: goat, biopsy needle, liver, laparoscopy

Recebido em 26 de maio de 2008

Aceito em 23 de dezembro de 2008

*Autor para correspondência (corresponding author)

E-mail: cattelan@fcav.unesp.br 


\section{INTRODUÇÃO}

A laparoscopia, técnica de invasão mínima, permite a inspeção da cavidade abdominal mediante a introdução de endoscópio rígido, denominado laparoscópio, o qual penetra a cavidade por meio de um trocarte. Com o intuito de diminuir risco de acidentes na penetração do trocarte, realiza-se, previamente, a infusão de ar ambiente (Silva et al., 1996) ou dióxido de carbono na cavidade peritonial, formando o que se denomina pneumoperitônio. Tal procedimento é realizado após punção com agulha de Veress, promovendo afastamento da parede abdominal em relação às vísceras, o que permite ampla visão ao operador (Silva et al., 2002).

A quantidade de gás a ser infundida está na dependência do porte do animal, sendo que a pressão ideal está relacionada à necessidade do procedimento (Silva et al., 2002). Cordeiro (2006), ao avaliar a laparoscopia na aspiração folicular em cabras, utilizou pressão intraabdominal calculada de acordo com o peso da fêmea, variando de 5 a $8 \mathrm{mmHg}$ e insuflação de manutenção de $2,5 \mathrm{~L} /$ minuto.

Em ruminantes, a biópsia hepática tem aplicação clínica importante, pois fornece aos profissionais informações sobre a estrutura morfológica e a composição detalhada do órgão (Amorim et al., 2003). Quando provas de função hepática denotam alteração na função do fígado, a determinação exata da lesão e de sua extensão somente poderá ser obtida com o uso de biópsia hepática (Silva et al., 2002).

Entre os animais, o porco é considerado o doador apropriado para humanos, por causa da semelhança em tamanho e função de seus órgãos. Porém, transplantes bem sucedidos de órgãos ou células de porco para humanos são limitados devido à ocorrência de rejeição hiperaguda. Assim, ao avaliar a aplicação de hepatócitos de caprinos como alternativa ao tratamento da falência hepática aguda em humanos, observouse que, para as funções citotóxicas, houve redução significante na viabilidade de hepatócitos suínos (38\%) quando comparada à de hepatócitos de caprinos e de fetos humanos, que retiveram sua viabilidade (98\%) em incubação com soro humano normal. Essas observações sugerem que hepatócitos de caprinos provavelmente possam ser empregados no tratamento da falência hepática aguda (Vijayalakshmi et al., 2004).

Em bovinos, técnicas de biópsia hepática por acesso percutâneo no $11^{\circ}$ espaço intercostal direito foram descritas por Braga et al. (1985) e Amorim et al. (2003), utilizando agulhas especiais. Ambas as técnicas permitiram a colheita de tecido hepático em todos os animais, e a amostra obtida foi considerada suficiente para exame histológico (Braga et al., 1985).

Tostes e Bandarra (2002) compararam biópsias hepáticas procedidas com agulhas aspirativa $\left(\right.$ Menghini $\left.^{\circledR}\right)$ e cortante $\left(\right.$ Tru-cut $\left.{ }^{\circledR}\right)$, utilizando técnica percutânea transabdominal em 60 cães portadores de afecções hepáticas. As amostras de tecido hepático colhidas com essas agulhas foram comparadas ao exame histológico postmortem do fígado desses animais, havendo maior concordância de acertos (88,3\%) com a agulha Tru-cut ${ }^{\circledR}$.

Estudo comparativo envolvendo pinça de biópsia de $5 \mathrm{~mm}$ e a agulha tru-cut em biópsias renais guiadas por laparoscopia foi realizado em 10 equinos hígidos, distribuídos aleatoriamente em dois grupos (Tabet et al., 2005). Os autores consideraram a biópsia renal com o uso de pinça como sendo a mais adequada, em virtude da obtenção de fragmentos maiores para exame histológico.

Nos animais de produção e trabalho, a biópsia hepática com pinça guiada por laparoscopia foi descrita em ovinos (Silva et al., 1996) e equinos (Silva et al., 2002), no entanto, estudos envolvendo caprinos são escassos. Este trabalho teve como objetivos descrever e avaliar a técnica de biópsia hepática em caprinos guiada por videolaparoscopia e, ainda, analisar, mediante exame histológico, os fragmentos hepáticos colhidos.

\section{MATERIAL E MÉTODOS}

Foram utilizados 12 caprinos machos, castrados, hígidos, sem raça definida, mantidos em baias onde tinham acesso a água, sal mineral, feno de coast cross (Cynodon dactylon) e silagem de milho à vontade, além de ração balanceada com $14 \%$ de proteína bruta fornecida uma vez ao dia (300g/animal). Conforme a idade, foram formados dois grupos experimentais, um com 
cinco caprinos de 12 meses de idade e pesos variando entre 20 e $36,4 \mathrm{~kg}(\mathrm{G} 1)$ e outro (G2) com sete animais de seis meses e pesos entre 14,6 e $19,6 \mathrm{~kg}$.

O manejo sanitário consistiu na limpeza diária das instalações e, mensalmente, foi feito o controle de endoparasitas por meio da verificação do número de ovos por grama de fezes (OPG) e da administração, quando necessária, do anti-helmíntico fenbendazole ${ }^{1}$ na dose de $5 \mathrm{mg} / \mathrm{kg}$ por via oral.

Durante a fase experimental, os animais foram submetidos a exames clínicos, e os dados de temperatura retal $\left({ }^{\circ} \mathrm{C}\right)$, frequências cardíaca e respiratória ( $\mathrm{n}^{\mathrm{o} / \text { minuto })} \mathrm{e}$ de movimentos rumenais ( $\mathrm{n}^{\mathrm{o}} /$ três minutos) obtidos nos períodos que antecederam o jejum (T0) e às 24 (T24), 48 (T48) e 72 (T72) horas após a biópsia hepática foram registrados para a análise estatística.

Após o jejum alimentar de 36 e hídrico de 24 horas, realizou-se a tricotomia do campo cirúrgico (flanco direito) e da região cervical para introdução de cateter na veia jugular direita, pelo qual foram aplicados os anestésicos. Todos os animais foram submetidos ao mesmo protocolo anestésico, que consistiu na administração, por via intramuscular, de $0,05 \mathrm{mg} / \mathrm{kg}$ de cloridrato de xilazina ${ }^{2}$ a $2 \%$. Decorridos 15 minutos, administrou-se $1 \mathrm{mg} / \mathrm{kg}$ de cloridrato de cetamina ${ }^{3}$ a $10 \%$, pela via intravenosa e, em sequência, os animais foram posicionados em decúbito lateral esquerdo, em maca cirúrgica apropriada. Introduziu-se um cateter $^{4}$ na veia jugular direita para manutenção anestésica pela administração, por infusão contínua, de anestesia total intravenosa (TIVA), na dose de $2 \mathrm{~mL} / \mathrm{kg} /$ hora. A solução empregada na TIVA foi constituída por soro fisiológico ${ }^{5}$ a $0,9 \%$ contendo $50 \mathrm{mg} / \mathrm{mL}$ de éter gliceril guaiacol ${ }^{6}, 0,05 \mathrm{mg} / \mathrm{mL}$ de cloridrato de xilazina e $1 \mathrm{mg} / \mathrm{mL}$ de cloridrato de cetamina, conforme descreve Riebold (1996).

${ }^{1}$ Panacur. Divisão Intervet. Akzo Nobel Ltda. - Cruzeiro, Brasil.

${ }^{2}$ Rompun. Bayer S.A. - São Paulo, Brasil.

${ }^{3}$ Cetamin. Syntec do Brasil Ltda. - Campinas, Brasil.

${ }^{4} \mathrm{BD}$ Insyte (14 GA x 45mm). Becton, Dickinson Ind. Cir. Ltda. - Juiz de Fora, Brasil.

${ }^{5}$ Cloreto de sódio a $0,9 \%$. JP Indústria Farmacêutica S.A. Ribeirão Preto, Brasil.

${ }^{6}$ Éter Gliceril Guaiacol. Henryfarma Ltda. - São Paulo, Brasil.
Após o início da infusão da TIVA, os animais foram submetidos à intubação oro-traqueal com sonda de Magill e auxílio de laringoscópio de lâmina reta. Uma vez intubado, administrou-se oxigênio num fluxo de $50 \mathrm{~mL} / \mathrm{kg} /$ minuto durante todo o procedimento cirúrgico.

Nos dois locais onde seriam realizadas as punções da cavidade abdominal para introdução do laparoscópio e da agulha de biópsia hepática, procedeu-se à anestesia local infiltrativa com $1 \mathrm{~mL}$ de cloridrato de lidocaína ${ }^{7}$ a $2 \%$ em cada local. As frequências cardíaca e respiratória e a temperatura retal de cada animal foram monitoradas continuamente durante toda a intervenção cirúrgica, até a recuperação completa da anestesia.

No procedimento cirúrgico, foi utilizado o material de rotina de diérese, hemostasia e síntese, além de equipamento de videolaparoscopia $^{8}$ composto por trocarte ${ }^{9}$ de $7 \mathrm{~mm}$, ótica rígida Hopkins ${ }^{10}$ de $7 \mathrm{~mm}$, insuflador $^{11}$, fonte de luz ${ }^{12}$ e microcâmera ${ }^{13}$ acoplada a monitor de vídeo ${ }^{14}$ por um cabo ótico $^{15}$.

Para a realização da laparoscopia, produziu-se previamente o pneumoperitônio com gás carbônico, conforme preconizado por Cordeiro (2006), mediante a introdução de cânula de Veress $^{16}$ no flanco direito, a aproximadamente $10 \mathrm{~cm}$ ventral aos processos transversos das vértebras lombares. Após a remoção da cânula de Veress, introduziu-se, pelo mesmo orifício, o trocarte e respectiva cânula no interior da cavidade abdominal. Em seguida, removeu-se o trocarte, e sua cânula permaneceu inserida no abdome para possibilitar a introdução da ótica rígida Hopkins do laparoscópio e permitir a visibilização intra-abdominal subsequente.

${ }^{7}$ Xylestesin. Cristália Prod. Quím. Farmacêuticos Ltda. Itapira, Brasil.

${ }^{8}$ Karl Storz Endoskope - Tuttlingen, Alemanha.

${ }^{9}$ Trocarte de $7 \mathrm{~mm} \mathrm{c}$ / entrada $\mathrm{p} / \mathrm{CO}_{2}$. Karl Storz - Tuttlingen, Alemanha.

${ }^{10}$ Ótica rígida Hopkins de $7 \mathrm{~mm} \mathrm{c} / 30^{\circ}$ inclinação. Karl Storz Tuttlingen, Alemanha.

${ }^{11}$ Electronic Endoflator. Karl Storz - Tuttlingen, Alemanha.

${ }^{12}$ Xenon Nova. Karl Storz - Tuttlingen, Alemanha.

${ }^{13}$ Endovision XL. Karl Storz - Tuttlingen, Alemanha.

${ }^{14}$ Sony Trinitron. Sony Corporation - Tóquio, Japão.

${ }^{15}$ Cabo de iluminação c/ fibra ótica. Karl Storz - Tuttlingen, Alemanha.

${ }^{16}$ Cânula para pneumoperitônio de Veress de $15 \mathrm{~cm}$. Edlo Produtos Médicos. Canoas, Brasil. 
Após a observação do fígado, introduziu-se a agulha de biópsia hepática ${ }^{17}$ (agulha tru-cut $14 \mathrm{G}$ x $11,4 \mathrm{~cm} \times 20 \mathrm{~mm}$ ) no $11^{\circ}$ espaço intercostal, a aproximadamente $12 \mathrm{~cm}$ ventral à coluna vertebral, direcionando-a ao órgão para punção e remoção de fragmento do lobo direito. Posteriormente à retirada do fragmento hepático, a hemorragia causada pela perfuração do órgão foi observada, e sua duração, em minutos, foi registrada.

Antes da remoção da cânula, o pneumoperitônio foi removido mediante massagem e compressão delicada do abdome. Em seguida, retirou-se a cânula e suturaram-se as duas incisões cutâneas com fio de náilon monofilamentar ${ }^{18} \mathrm{n}^{\mathrm{o}} 0 \mathrm{com}$ um ponto simples separado por incisão.

Os fragmentos hepáticos colhidos foram fixados em solução de Bouin por 24 horas, sendo processados de forma rotineira para inclusão em parafina e obtenção de cortes de seis micrômetros. Os cortes foram corados pela hematoxilina-eosina, conforme Behmer et al. (1976). Diariamente, as feridas operatórias foram limpas e os curativos feitos com povidona-iodo ${ }^{19}$ até a cicatrização completa.

Para a descrição dos achados clinico-cirúrgicos e histológicos, utilizaram-se as percentagens de ocorrências. Para os dados obtidos nas avaliações da temperatura retal, das frequências cardíaca e respiratória e dos movimentos rumenais, empregou-se o delineamento inteiramente ao acaso, com medidas repetidas no tempo (2 grupos e 4 momentos). Empregou-se a análise de variância e, em seguida, o teste Tukey para a comparação das médias segundo procedimentos do General Linear Models (Proc GLM) do SAS/STAT/1999, considerando os valores significativos quando $\mathrm{P} \leq 0,05$.

\section{RESULTADOS E DISCUSSÃO}

O tempo de 36 e 24 horas de jejum alimentar e hídrico, respectivamente, foi considerado adequado para a execução do procedimento cirúrgico, não sendo observadas distensão visceral e regurgitação de alimentos durante a

\footnotetext{
${ }^{17}$ Tru-cut Biopsy Needle (14G x 11,4cm x 20mm). Allegiance Healthcare Corp. Oklahoma - EUA.

${ }^{18}$ Mononylon. Ethicon - São José dos Campos, Brasil.

${ }^{19}$ Riodeíne. Rioquímica - São José do Rio Preto, Brasil.
}

manobra de biópsia. Essa última complicação foi relatada em ovinos submetidos à laparoscopia após jejum alimentar de 72 e hídrico de 24 horas (Bittencourt et al., 2004). Os autores atribuíram a regurgitação de conteúdo rumenal ao pneumoperitônio e à anestesia intravenosa pela cetamina e acepromazina.

Os animais foram intubados com sonda de Magill, de calibres variáveis, entre 4,5 e 6,0 , de acordo com o tamanho do animal. Esse procedimento foi adotado para evitar a aspiração de saliva pelos órgãos respiratórios, pois todos os animais apresentaram sialorréia durante a indução anestésica. Bittencourt et al. (2004) observaram sialorréia no período de recuperação anestésica de ovelhas submetidas a procedimentos videolaparoscópicos e atribuíramna à cetamina, que também foi empregada neste experimento.

O procedimento anestésico empregado nesta pesquisa, conforme descrito por Riebold (1996) e associado à anestesia local infiltrativa, foi considerado apropriado, pois durante a intervenção cirúrgica nenhum animal apresentou, clinicamente, sinais de dor, o que também foi observado por Cordeiro (2006).

Para produção do pneumoperitônio, foi utilizada pressão intra-abdominal de 7 a $9 \mathrm{mmHg}$, conforme o tamanho do animal, assim como recomendaram Tabet et al. (2005) e Cordeiro (2006). Essa pressão intra-abdominal foi considerada suficiente para afastar a parede abdominal das vísceras, permitindo ao operador a visibilização apropriada da cavidade abdominal e de seus órgãos.

Em um animal do G2, houve perfuração do cólon espiral durante a introdução da cânula de Veress e, devido a esse acidente, administrou-se associação de penicilinas ${ }^{20}$ na dose de 30.000UI de penicilina benzatina $/ \mathrm{kg}$ a cada 48 horas, por via intramuscular, totalizando três aplicações. Não foram verificadas anormalidades em sua recuperação. Tabet et al. (2005) relataram que possíveis acidentes trans-operatórios podem ser observados, em especial a laceração de vísceras, no momento de introdução do trocarte. Contudo, não há relatos de enterocentese acidental com o

\footnotetext{
${ }^{20}$ Pentabiótico pequeno porte, Fort Dodge Saúde Animal Ltda. - Campinas, Brasil.
} 
uso da cânula de Veress. Possivelmente, a perfuração intestinal tenha sido causada pelo posicionamento incorreto dessa cânula de comprimento relativamente longo $(15 \mathrm{~cm})$ associado à perfuração abrupta da parede abdominal.

A hemorragia provocada no fígado pelo procedimento de biópsia foi observada até cessar, o que ocorreu aos dois minutos em $75 \%$ dos animais (quatro do G1 e cinco do G2) e em três minutos nos outros $25 \%$ (um do G1 e dois do
G2). Estes achados ratificam os de Silva et al. (2002), que observaram sangramento hepático mínimo com o uso de pinça de biópsia em equinos. Confirmando as informações de Nord (1992) e Fantinatti et al. (2003), a hemorragia proveniente de biópsia hepática é frequente, mas a observada nesta pesquisa foi considerada clinicamente irrelevante, a julgar pela evolução pós-operatória favorável e sem alterações dos parâmetros avaliados no exame físico (Tab. 1) de todos os animais.

Tabela 1. Médias e desvios-padrão dos valores de temperatura retal (TR), frequência cardíaca (FC), frequência respiratória (FR) e de movimentos rumenais (MR) segundo os grupos, no pré-jejum (T0) e às 24 (T24), 48 (T48) e 72 (T72) horas após a biópsia hepática em caprinos pelo acesso laparoscópico

\begin{tabular}{|c|c|c|c|c|}
\hline & \multicolumn{4}{|c|}{ Momento } \\
\hline & T0 & $\mathrm{T} 24$ & $\mathrm{~T} 48$ & $\mathrm{~T} 72$ \\
\hline & \multicolumn{4}{|c|}{$\operatorname{TR}\left({ }^{\circ} \mathrm{C}\right)$} \\
\hline G1 & $38,86 \pm 0,32$ & $39,22 \pm 0,77$ & $39,06 \pm 0,53$ & $39,28 \pm 0,59$ \\
\hline G2 & $38,76 \pm 0,35$ & $39,40 \pm 0,60$ & $39,28 \pm 0,73$ & $38,94 \pm 0,41$ \\
\hline \multirow[t]{2}{*}{ Geral (G1+G2) } & $38,72 \pm 0,32$ & $39,32 \pm 0,64$ & $39,19 \pm 0,63$ & $39,08 \pm 0,50$ \\
\hline & \multicolumn{4}{|c|}{$\mathrm{FC}(\mathrm{n} / \mathrm{min})$} \\
\hline G1 & $84,40 \pm 21,09$ & $105,60 \pm 17,30$ & $92,00 \pm 9,56$ & $85,60 \pm 8,62$ \\
\hline G2 & $82,00 \pm 12,53$ & $83,86 \pm 7,49$ & $92,14 \pm 11,47$ & $82,58 \pm 11,77$ \\
\hline \multirow[t]{2}{*}{ Geral (G1+G2) } & $83,00 \pm 16,16$ & $92,92 \pm 16,27$ & $92,08 \pm 10,25$ & $83,83 \pm 10,25$ \\
\hline & \multicolumn{4}{|c|}{$\mathrm{FR}(\mathrm{n} / \mathrm{min})$} \\
\hline G1 & $34,38 \pm 9,09$ & $30,80 \pm 9,20$ & $28,60 \pm 9,45$ & $31,00 \pm 13,49$ \\
\hline G2 & $29,43 \pm 3,55$ & $32,71 \pm 10,80$ & $28,71 \pm 5,65$ & $27,00 \pm 4,32$ \\
\hline \multirow[t]{2}{*}{ Geral $(\mathrm{G} 1+\mathrm{G} 2)$} & $29,82 \pm 6,20$ & $31,92 \pm 9,76$ & $28,67 \pm 7,06$ & $28,67 \pm 8,98$ \\
\hline & \multicolumn{4}{|c|}{$\mathrm{MR}(\mathrm{n} / 3 \mathrm{~min})$} \\
\hline G1 & $2,40 \pm 0,55$ & $2,00 \pm 0,71$ & $1,80 \pm 0,84$ & $2,40 \pm 0,55$ \\
\hline $\mathrm{G} 2$ & $2,28 \pm 0,49$ & $2,00 \pm 0,15$ & $2,00 \pm 0,58$ & $2,28 \pm 0,49$ \\
\hline Geral (G1+G2) & $2,36 \pm 0,49$ & $2,00 \pm 0,43$ & $1,92 \pm 0,67$ & $2,33 \pm 0,49$ \\
\hline
\end{tabular}

G1: animais com 12 meses de idade; G2: animais com seis meses de idade.

Não houve diferença entre as médias pelo teste Tukey $(\mathrm{P}>0,05)$.

O local escolhido para a introdução da agulha tru-cut e punção do lobo hepático direito, correspondente ao $11^{\circ}$ espaço intercostal direito, embasou-se nas descrições feitas para punção hepática percutânea em bovinos (Braga et al., 1985; Amorim et al., 2003) e permitiu que a punção do órgão fosse realizada com precisão e segurança.

Com a visibilização do fígado, pôde-se ter confiança e assegurar a biópsia do órgão alvo em $100 \%$ dos animais, sendo posteriormente essa observação confirmada pela análise histológica. Similarmente às observações desta investigação, a segurança do procedimento de biópsia hepática guiada por laparoscopia já fôra relatada em ovinos (Silva et al., 1996), equinos (Silva et al., 2002) e caninos (Fantinatti et al., 2003). Entretanto, a técnica cirúrgica empregada nesta pesquisa difere da relatada em ovinos por Silva et al. (1996), que introduziram a pinça de biópsia pelo interior do laparoscópio inserido no flanco direito, no terço médio entre os processos transversos das vértebras lombares e a linha alba, caudal à última costela.

O tempo operatório médio foi de 23 minutos e cinco segundos (tempos operatórios em numerais ordinais: $31,27,19,30,22,13,30,25,23,18,19$ e 20 minutos). A experiência e a capacitação do 
cirurgião ao longo dos procedimentos laparoscópicos permitiram que esse tempo fosse reduzido com o desenvolvimento do experimento (26min e $45 \mathrm{seg} \pm 5 \mathrm{~min}$ e $26 \mathrm{seg}$, nos quatro primeiros; $20 \mathrm{~min} \pm 2 \mathrm{~min}$ e $10 \mathrm{seg}$, nos quatro últimos animais).

Os animais não receberam antibióticos, antiinflamatórios ou analgésicos após o procedimento e, assemelhando-se às observações de Tabet et al. (2005) após a execução de biópsias renais em equinos, não foram observados sinais de infecção ou desconforto no período pós-operatório. Somente um animal foi submetido à antibioticoterapia sistêmica, conforme descrito previamente.

$\mathrm{Na}$ análise estatística, observou-se que os valores do teste $\mathrm{F}$ para grupo, momento e da interação grupo e momento não apresentaram diferença significativa $(\mathrm{P}>0,05)$. As médias e respectivos desvios-padrão da temperatura retal e das frequências cardíaca, respiratória e dos movimentos rumenais constam da Tab. 1 e, de acordo com Pugh (2005), os dados observados são considerados físiológicos para a espécie caprina.

O fragmento hepático obtido pela agulha de biópsia tru-cut (Fig. 1A) foi considerado suficiente para a avaliação histológica. Nas preparações histológicas examinadas, observouse tecido hepático normal em $100 \%$ dos animais, confirmando as descrições de Banks (1991), Young e Heath (2001) e Bacha Júnior e Bacha (2003).

Considerando-se o lóbulo hepático (Banks, 1991) composto por uma veia centrolobular circundado por tríades portais (ramificação da artéria hepática, ramificação da veia hepática e um ducto biliar), nas lâminas estudadas, foram observadas um máximo de três tríades por lóbulo hepático. A distância entre as veias centrolobulares dos lóbulos foi, em $66,7 \%$ dos animais (três do G1 e cinco do G2), maior do que o diâmetro da amostra obtida no experimento por consequência da largura da agulha de biópsia (Fig. 1B). Os lóbulos portais, definidos por um ducto biliar ao centro de três veias centrolobulares, também foram vistos parcialmente, em função de o espaço entre as referidas veias ser maior do que o observado nas amostras de biópsia hepática obtidas.

Os ácinos hepáticos, divisão funcional do fígado composta por duas tríades e partindo da vascularização entre elas em direção às veias centrolobulares (Young e Heath, 2001), foram observados na maioria dos cortes histológicos. A visualização desses ácinos foi mais bem efetuada quando as veias que os compunham estavam seguindo a orientação do corte estudado, o que ocorreu em $75 \%$ (quatro animais do G1 e cinco do G2) das preparações examinadas. Foram observados hepatócitos, células de Kupfer e células endoteliais. Os hepatócitos apresentaramse com formato poliédrico e dispostos em cordões celulares partindo radialmente das veias centrolobulares em direção à periferia do lóbulo hepático. Não foram observadas divisões de tecido conjuntivo entre os lóbulos hepáticos, o que é normal em ruminantes e, assim, o limite entre os lóbulos foi considerado a partir do desencontro observado entre os sinusoides de lóbulos diferentes, conforme descrito por Banks (1991) e Bacha Júnior e Bacha (2003). Todos os sinusoides observados apresentaram-se bem arranjados, sem espaçamentos ou infiltrações celulares dignas de nota, confirmando a descrição de Banks (1991).

O procedimento de biópsia hepática com agulha tru-cut, conforme executado nesta pesquisa, pode permitir colheitas adicionais de tecido hepático para exame histológico, inclusive em animais com lesões localizadas, propiciando que o tecido alterado seja avaliado e comparado ao proveniente de áreas normais. Por isso, supõe-se que esta técnica poderá contribuir decisivamente no diagnóstico, prognóstico e acompanhamento do tratamento instituído em caprinos com afecções hepáticas. Ao comparar biópsias hepáticas procedidas com agulhas aspirativa $\left(\right.$ Menghini ${ }^{\circledR}$ ) e cortante $\left(\right.$ Tru-cut $\left.{ }^{\circledR}\right)$, utilizando técnica percutânea transabdominal em cães, Tostes e Bandarra (2002) observaram índice de acerto maior $(88,3 \%)$ com o uso de agulha cortante. Concluíram que a agulha tru-cut permite diagnósticos corretos independentemente da natureza da lesão, devido à estreita relação entre o volume maior das amostras obtidas com essa agulha de biópsia e a maior possibilidade de acerto do diagnóstico. 


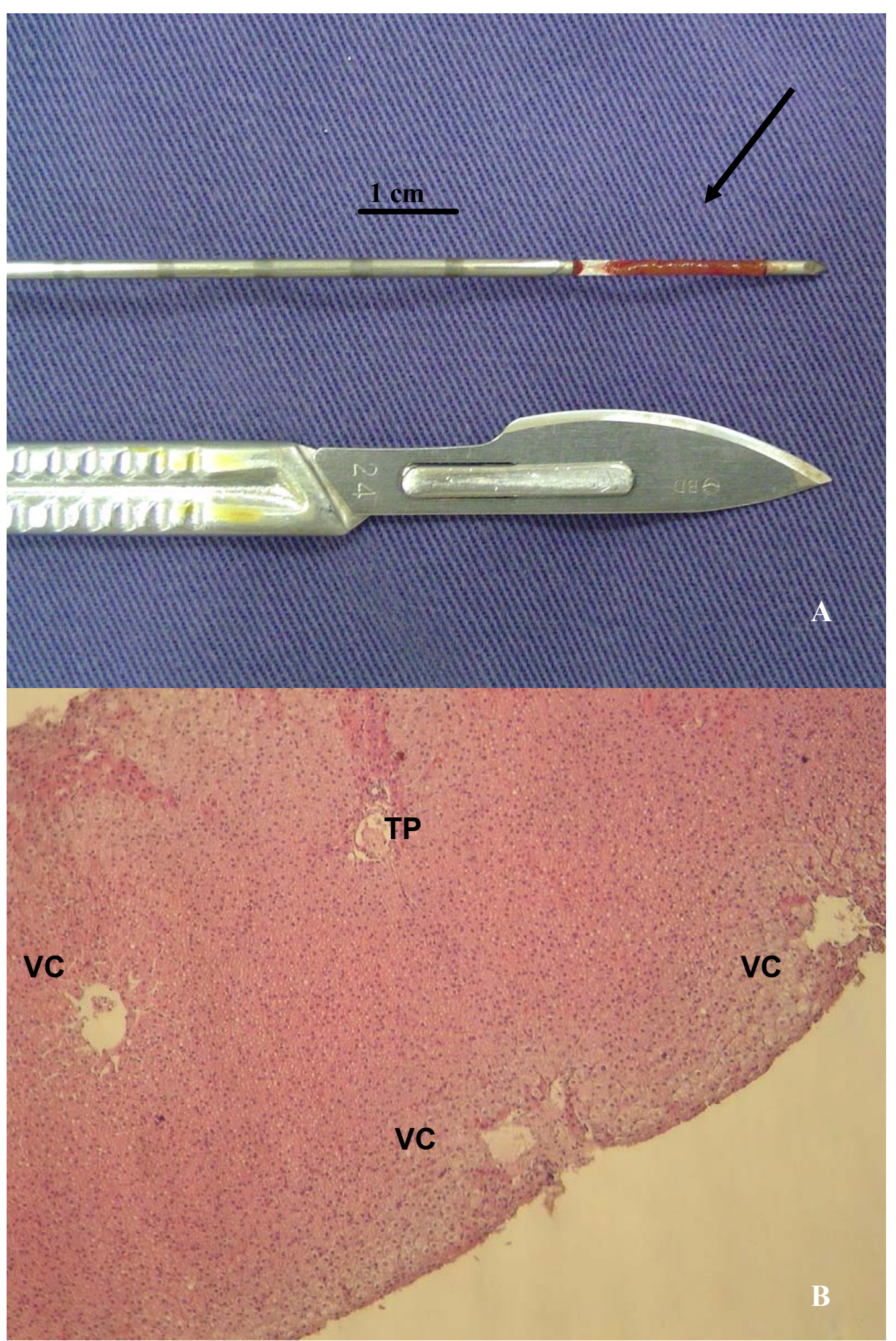

Figura 1. A: Imagem fotográfica ilustrando o fragmento hepático (seta) obtido com agulha tru-cut; B: Fotomicrografia de tecido hepático de caprino obtido por biópsia videolaparoscópica com agulha tru-cut. Notar as veias centrolobulares (VC) e a tríade portal (TP), formando o lóbulo portal. HE. Objetiva 10X. 
Acredita-se que esse procedimento poderá ser aplicado, entre outros fins, para estudos diversos de cultivo in vitro de fígado, bem como para a elaboração de testes in vitro de compatibilidade futura para transplantes de tecidos entre indivíduos da mesma espécie (alotransplante) ou de espécies diferentes (xenotransplantes), conforme relataram Vijayalakshmi et al. (2004).

\section{CONCLUSÃo}

A biópsia hepática com agulha tru-cut guiada por videolaparoscopia é eficaz para uso em caprinos e permite a obtenção de fragmentos hepáticos suficientes para exame histológico.

\section{AGRADECIMENTOS}

Os autores agradecem o apoio financeiro da Fundação de Amparo à Pesquisa do Estado de São Paulo (FAPESP) e a colaboração do Prof. Dr. Euclides Braga Malheiros na análise estatística.

\section{REFERÊNCIAS BIBLIOGRÁFICAS}

AMORIM, R.M.; BORGES, A.S.; KUCHEMBUCK, M.R.G. et al. Bioquímica sérica e hemograma de bovinos antes e após a técnica de biópsia hepática. Cienc. Rural, v.33, p.519-523, 2003.

BACHA JÚNIOR, W.J.; BACHA, L.M. Atlas colorido de histologia veterinária. 2.ed. São Paulo: Roca, 2003. p.197.

BANKS, W.J. Histologia veterinária aplicada. 2.ed. São Paulo: Manole, 1991. p.468-478.

BEHMER, O.A.; TOLOSA, E.M.C.; FREITAS NETO, A.G. Manual de técnicas para histologia normal e patológica. São Paulo: Edart, 1976. $256 \mathrm{p}$.

BITTENCOURT, R.H.F.P.M.; SILVA, M.C.; MOREIRA, V.M.S. et al. Observações sobre o uso de cetamina, acepromazina e halotano em ovelhas submetidas a procedimentos laparoscópicos. Rev. Bras. Med. Vet., v.26, p.150-154, 2004.

BRAGA, M.M.; CASTILHOS, L.M.L.; SANTOS, M.N. Biópsia hepática em bovinos: proposta de nova técnica. Rev. Cent. Cienc. Rurais, v.15, p.79-88, 1985.
CORDEIRO, M.F. Avaliação da laparoscopia na aspiração folicular em fêmeas caprinas prépúberes e adultas com ou sem estimulação ovariana hormonal. 2006. 59f. Tese (Doutorado) - Faculdade de Ciências Agrárias e Veterinárias, Universidade Estadual Paulista, Jaboticabal.

FANTINATTI, A.P.; DALECK, C.R.; NUNES, N. et al. Laparoscopy hepatic biopsy through cauterization. Cienc. Rural, v.33, p.703-707, 2003.

NORD, H.J. Complications of laparoscopy. Endoscopy, v.24, p.693-700, 1992.

PUGH, D.G. Clínica de ovinos e caprinos. São Paulo: Roca, 2005. p.1-15.

RIEBOLD, T.W. Ruminants. In: THURMON, J.C.; TRANQUILLI, W.J.; BENSON, G.J. Lumb \& Jones' veterinary anesthesia. 3.ed. Philadelphia: Lea \& Febiger, 1996. p.613-614.

SILVA, L.C.L.C.; STOPIGLIA, A.J.; FANTONI, D.T. Técnica de biópsia hepática em eqüino por laparoscopia. Cienc. Rural, v.32, p.459-465, 2002.

SILVA, L.C.L.C.; FERREIRA, M.A.; GOMEZ, H.M. et al. Biópsia hepática em ovinos por via laparoscópica. In: CONGRESSO BRASILEIRO DE CIRURGIA E ANESTESIOLOGIA VETERINÁRIA, 2., 1996, Ribeirão Preto. Anais... Santa Maria: CBCAV, 1996. p.108-109. (Resumo).

TABET, A.F.; SILVA, L.C.L.C.; SHINHORINI, I.L. et al. Comparação entre duas técnicas de biópsia renal guiadas por laparoscopia em eqüinos. Braz. J. Vet. Res. Anim. Sci., v.42, p.150-156, 2005.

TOSTES, R.A.; BANDARRA, E.P. Biopsia hepática em cães: relação entre qualidade da amostra e grau de conclusão do diagnóstico. Arq. Bras. Med. Vet. Zootec., v.54, p.468-472, 2002.

VIJAYALAKSHMI, V.; NASEEM, B.; KHAN, A.A. et al. Comparison of biochemical and citotoxic functions of hepatocytes from goat, pig and human fetuses. J. Gastroenterol. Hepatol., v.19, p.1029-1035, 2004.

YOUNG, B.; HEATH, J.W. Histologia funcional: texto e atlas em cores. 4.ed. Rio de Janeiro: Guanabara Koogan, 2001. p.274-278. 